\title{
Artificial Generation of Wind and Estimation of the Spectrum of the Wind from Structural Response
}

\author{
Junko Minato*, Akira Ohsumi**, and Yuichi Sawada* \\ * Department of Mechanical and System Engineering, Graduate School of Science and Technology, \\ Kyoto Institute of Technology \\ Matsugasaki, Sakyo, Kyoto 606-8585, Japan \\ E-mail: \{joy73101, sawada\}@kit.ac.jp \\ ** Professor Emeritus of Kyoto Institute of Technology \\ E-mail: akiraspika@nifty.com
}

\begin{abstract}
A method of generating artificial wind load is proposed incorporating with the recommendations for statistics of the random wind offered in the architecture community. The method consists of two steps; the first one is to generate the wind velocity from the computer simulated white noise, and the second is to produce the wind pressure from the wind velocity using the aerodynamic admittance. An inverse problem is further investigated for estimating wind load acting on the building structure from the measurement data which is made on the response of the structure.
\end{abstract}

\section{Introduction}

It will be commonly believed that one of important challenges in structural design is the generation of artificial wind loads and/or seismic waves. These loads or waves are necessary in computer simulations to show how well the structure withstands vibrations caused by these actions. Especially, for the analysis of random responses of structures such as long-span bridges, highrise buildings and space structures (which are called flexible structures in control system engineering), one needs, besides the spectral response, the time-history of the seismic waves and/or wind loads. Of course, there are so many recorded data on the real seismic waves and wind velocity or pressure. Needless to say, however, the models to generate such waves or loads artificially are still required to investigate the structural responses. As for the seismic waves, one of the authors has presented a model based on the chaplet-based signal approximation and the Wigner-Ville distribution, and applied it to reproduce the real seismic waves [1].

In this paper the authors concentrate their attentions to the artificial wind loads. Although the significance of artificial wind has been recognized quite a while, much more in-depth research is necessary before any meaningful engineering design conclusions can be reached. The researches on the statistical nature of the wind have a long history (e.g., see [2]-[7]). However, there have been very few studies that tried to propose the method of generating artificial wind load in spite of its significance.

For instance, Iwatani [8], [9] proposed a method of generating wind fluctuations whose power and cross spectral densities become to coincide with the given desirable ones. His approach is to use the vector autoregressive (AR) model for describing the wind fluctuation and to determine coefficients of the model in such a way that the output of the AR model satisfy the given power and cross spectra. The variances of the AR model are computed after the coefficient matrices have been determined. This approach seems to require numerous computations for solving simultaneous equations. Recently, the authors [10] have proposed two methods for generating random wind velocity whose power and cross spectral densities are satisfied by reflecting actual winds. The one is based on the linear dynamic system with white noise process as the seed of randomness, while the other is derived based on the spectral representation of a stationary process in which the orthogonal process is modeled by the Wiener process on the frequency domain.

Our purpose in this paper is twofold: Firstly, a method for generating wind loads is developed from the first method mentioned above with numerical results; and then, using such wind loads generated artificially, a possibility is probed to solve an inverse problem how to estimate the wind load acting on a high-rise building from the measurement data which are made on the vibration of the building.

The paper is organized as follows. In Section 2 a method for generating vector wind loads from the computer-simulated white noise processes is proposed by a two-step procedure. First, the spectral density of the wind velocities at whole stories of the building structure is modeled to satisfy the given power and cross power densities. Then, the spectral density of the windward pressure is calculated from the density obtained for the wind velocities. The overall fre- 
quency transfer function is derived by incorporating the aerodynamic admittance. Fourier-inverting this transfer function, the impulse response matrix is obtained. Thus, using this impulse response, the vector wind load having desired statistical nature is generated by white noise processes as the inputs. An inverse problem of estimating the unknown wind load is investigated in Section 3. Simulation studies are given in Section 4 to show how well the wind loads are generated artificially and to see how we can estimate the spectrum of the wind loads from the response data of the building structure. Section 5 concludes the paper.

\section{Generation of Wind Load}

\subsection{Approach}

Suppose an $n$-story high-rise building. The wind load is evaluated by the windward pressure acting on the tributary area of the building. Let $P_{i}(t)(i=1,2, \cdots, n)$ be the wind pressure at the height $h_{i}$ of the $i$ th floor and expressed as

$$
P_{i}(t)=\bar{P}_{i}+p_{i}(t)
$$

where $\bar{P}_{i}$ is the mean pressure and $p_{i}(t)$ is the random fluctuation of the pressure. The mean $\bar{P}_{i}$ is given by the model,

$$
\bar{P}_{i}=\frac{1}{2} a_{i} C_{D i} \rho \bar{V}_{i}^{2}
$$

where $a_{i}$ is the tributary area for the $i$ th story unit; $C_{D i}$ the windward drag coefficient at $h_{i} ; \rho$ the air density; and $\bar{V}_{i}$ is the average wind velocity given below.

Invoking that the white noise can be easily generated by a digital computer, we use it as a seed of randomness for the wind load. Then, it will be conventional to generate $p_{i}(t)$ as an output of a linear system whose inputs are a set of white Gaussian noise processes $\left\{w_{k}(t)\right\}_{k=1,2, \cdots, n}$. To fix the idea, write this as

$$
\begin{aligned}
p_{i}(t) & =\sum_{k=1}^{n} \int_{-\infty}^{t} h_{p_{i} w_{k}}(t-\tau) w_{k}(\tau) d \tau \\
& =\sum_{k=1}^{n} \int_{0}^{\infty} h_{p_{i} w_{k}}(\tau) w_{k}(t-\tau) d \tau
\end{aligned}
$$

where $h_{p_{i} w_{k}}(t)$ is the impulse response function from $w_{k}(t)$ to $p_{i}(t)$. Or, in a vector form,

$$
p(t)=\int_{0}^{\infty} H_{p w}(\tau) w(t-\tau) d \tau,
$$

where $p(t)=\left[p_{1}(t), \cdots, p_{n}(t)\right]^{T}, w(t)=\left[w_{1}(t), \cdots\right.$, $\left.w_{n}(t)\right]^{T}$ ( $T$ denotes transpose), and $H_{p w}(t)$ is the $n \times n$-impulse response matrix, $H_{p w}(t)=$ $\left[h_{p_{i} w_{k}}(t)\right]_{i, k=1,2, \cdots, n}$. Equation (4) is the basic equation for generating the wind pressure $p(t)$ artificially from the computer-generated white noise process $w(t)$.
Recalling that the impulse response of a linear system is given by the inverse Fourier transform of the frequency transfer function, we have the relation,

$$
H_{p w}(t)=\mathcal{F}^{-1}\left[G_{p w}(j \omega)\right],
$$

so that the problem is how to design the frequency transfer function matrix $G_{p w}(j \omega)$ from $w(t)$ to $p(t)$ in which $j=\sqrt{-1}$.

Due to the lack of theories on the direct design of $G_{p w}(j \omega)$, unfortunately, the straightforward use of (4) is quite unrealistic. Alternatively, to exploit the accumulated knowledge or guidelines in the architecture community, we take two steps. First, according to the AIJ (Architectural Institute of Japan) Recommendations on the statistical properties of wind velocity denoted by $v(t)$, the power spectral density of the wind velocity, $S_{v}(\omega)$, is modeled, and then the power spectral density of the wind pressure, $S_{p}(\omega)$, is related to $S_{v}(\omega)$ using the aerodynamic admittance (the subscripts $v$ and $p$ stand for velocity and pressure). By assuming the wind velocity is generated by the simulated white noise, the wind pressure $p(t)$ is generated indirectly from the white noise through the wind velocity. Schematically, the artificial generation of the wind pressure $p(t)$ is illustrated in Fig. 1.

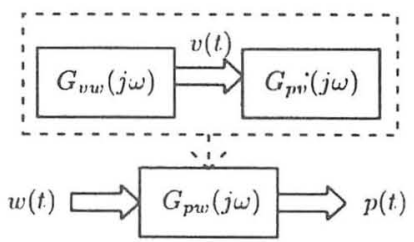

Fig. 1: Generation of the wind pressure $p(t)$ from the computer-generated white noise $w(t)$ whose spectral density $S_{w}(\omega)$ is constant.

Obviously, it is known from Fig. 1 that the transfer function matrix $G_{p w}(j \omega)$ is obtained in the following manner:

$$
G_{p w}(j \omega)=G_{p v}(j \omega) G_{v w}(j \omega)
$$

\subsection{Spectral Density of Wind Velocity}

Let $V_{i}(t)$ be the wind velocity at the height $h_{i}$ and be expressed similarly to (1) as

$$
V_{i}(t)=\bar{V}_{i}+v_{i}(t)
$$

where $\bar{V}_{i}$ is the mean (which is measured as the average during ten minutes), and $v_{i}(t)$ is the random fluctuation having variance $\sigma_{i}^{2}$. The statistical mean of $v_{i}(t)$ is zero. For $\bar{V}_{i}$ the model is recommended [11], [12]:

$$
\bar{V}_{i}=\bar{V}_{r}\left(\frac{h_{i}}{h_{r}}\right)^{\alpha}
$$

where $h_{r}$ and $\bar{V}_{r}$ are the reference height and average wind velocity at $h_{r}$, respectively; and the exponent $\alpha$ is recommended to take between 0.15 and 0.5 . 
Here, let $v(t)=\left[v_{1}(t), \cdots, v_{n}(t)\right]^{T}$ and let $S_{v}(\omega)$ be the spectral density matrix for the $v(t)$-process. Assume that $\left\{v_{i}(t)\right\}$ are correlated mutually (i.e., spatially). Then, its $(i, k)$-component of $S_{v}(\omega)=$ $\left[S_{v_{i} v_{k}}(\omega)\right]_{i, k=1,2, \cdots, n}$ is given by

$$
S_{v_{i} v_{k}}(\omega)=\left\{\begin{array}{cc}
S_{v_{i}}(\omega) & (i=k) \\
\sqrt{S_{v_{i}}(\omega)} \sqrt{S_{v_{k}}(\omega)} \operatorname{coh}_{i k}(\omega) & (i \neq k),
\end{array}\right.
$$

where $S_{v_{i}}(\omega)$ is the spectral density for $v_{i}(t)$-process, and $\operatorname{coh}_{i k}(\omega)$ is the coherence defined as the square root of

$$
\operatorname{coh}_{i k}^{2}(\omega)=\frac{\left|S_{v_{i} v_{k}}(\omega)\right|^{2}}{S_{v_{i}}(\omega) S_{v_{k}}(\omega)} .
$$

The coherence is a complex function of $\omega$, and can be interpreted as the phase difference of the two power spectra $S_{v_{i}}(\omega)$ and $S_{v_{k}}(\omega)$. Indeed, to show this, denote by $V_{i}(\omega)$ the Fourier transform of $v_{i}(t)$, i.e., $V_{i}(\omega)=\mathcal{F}\left[v_{i}(t)\right]$. Then, it is well-known that for sample processes $v_{i}(t)$ and $v_{k}(t)$ on a finite time interval $[-T / 2, T / 2]$ the cross spectral density $S_{v_{i} v_{k}}(\omega)$ can be expressed as

$$
S_{v_{i} v_{k}}(\omega)=\lim _{T \rightarrow \infty} \frac{1}{T} V_{i}(\omega) V_{k}^{*}(\omega)
$$

where the asterisk denotes the complex conjugate transpose. Here, write $V_{i}(\omega)$ as

$$
V_{i}(\omega)=\left|V_{i}(\omega)\right| e^{j \theta_{i}(\omega)},
$$

where $\left|V_{i}(\omega)\right|$ is the magnitude spectrum and $\theta_{i}(\omega)$ is the phase spectrum. So, (9) can be rewritten as follows:

$$
\begin{aligned}
S_{v_{i} v_{k}}(\omega) & =\lim _{T \rightarrow \infty} \frac{\left|V_{i}(\omega)\right|}{\sqrt{T}} \frac{\left|V_{k}(\omega)\right|}{\sqrt{T}} e^{j \theta_{i k}(\omega)} \\
& \equiv \sqrt{S_{v_{i}}(\omega)} \sqrt{S_{v_{k}}(\omega)} \operatorname{coh}_{i k}(\omega),
\end{aligned}
$$

where $\theta_{i k}(\omega)=\theta_{i}(\omega)-\theta_{k}(\omega)$. This relation shows that the coherence is just the phase difference of the two spectra $S_{v_{i}}(\omega)$ and $S_{v_{k}}(\omega)$.

According to the AIJ Recommendations, the model for $S_{v_{i}}(\omega)$ is given by [12]

$$
S_{v_{i}}(\omega)=\frac{0.238}{b_{i}\left\{1+\left(\frac{\omega}{2 \pi b_{i}}\right)\right\}^{5 / 6} \sigma_{i}^{2}}
$$

in which $\sigma_{i}^{2}$ is the variance of $v_{i}(t)$, and

$$
b_{i}=\frac{\bar{V}_{i}}{\ell\left(h_{i}\right)} \quad\left(\ell\left(h_{i}\right): \text { fluctuation length }\right) .
$$

It is known that the real part of the coherence is called the co-coherence. Write it as $\operatorname{cocoh}_{i k}(\omega)$, i.e., $\operatorname{cocoh}_{i k}(\omega)=\operatorname{Re}\left\{\operatorname{coh}_{i k}(\omega)\right\}$. Then, the relation

$$
\operatorname{coh}_{i k}(\omega)=\operatorname{cocoh}_{i k}(\omega)+j \sqrt{1-\operatorname{cocoh}_{i h}^{2}(\omega)}
$$

is obvious. The AIJ Recommendation for the cocoherence is [12]

$$
\operatorname{cocoh}_{i k}(\omega)=\exp \left\{-k_{0} \frac{\omega}{2 \pi} \frac{\left|h_{i}-h_{k}\right|}{\bar{V}_{r}}\right\},
$$

where $k_{0}$ is the decay factor, and $\bar{V}_{r}$ is the same reference mean wind velocity as used in (8).

Therefore, the matrix $S_{v}(\omega)=\left[S_{v_{i} v_{k}}(\omega)\right]_{i, k=1,2, \cdots, n}$ is expressed as follows:

$$
\begin{aligned}
S_{v}(\omega) & =\left[\sqrt{S_{v_{i}}(\omega)} \operatorname{coh}_{i k}(\omega) \sqrt{S_{v_{k}}(\omega)}\right]_{i, k=1,2, \cdots, n} \\
& =\sqrt{S_{v}(\omega)} C(\omega) \sqrt{S_{v}(\omega)},
\end{aligned}
$$

where the notation $\sqrt{S_{v}(\omega)}$ is defined by $\sqrt{S_{v}(\omega)}=$ $\operatorname{diag}\left\{\sqrt{S_{v_{1}}(\omega)}, \cdots, \sqrt{S_{v_{n}}(\omega)}\right\}$, and

$$
C(\omega)=\left[\operatorname{coh}_{i k}(\omega)\right]_{i, k=1,2, \cdots, n} .
$$

\subsection{Spectral Density of Wind Pressure}

Referring to Fig. 1, we have the relation between spectral densities $S_{p}(\omega)$ and $S_{v}(\omega)$ :

$$
S_{p}(\omega)=G_{p v}(j \omega) S_{v}(\omega) G_{p v}^{*}(j \omega)
$$

in which $S_{v}(\omega)$ has been obtained in the previous subsection. The transfer function matrix $G_{p v}(j \omega)$ can be determined from the aerodynamic admittance as follows:

$$
\left[G_{p v}(j \omega)\right]_{i k}=\left\{\begin{array}{cc}
C_{D i} \rho a_{i} \bar{V}_{i} \chi_{i}(\omega) & (k=i) \\
0 & (k \neq i),
\end{array}\right.
$$

where $\chi_{i}(\omega)$ is the aerodynamic admittance given by

$$
\chi_{i}(\omega)=\left[1+\left(j \omega \frac{\sqrt{a_{i}}}{\pi \bar{V}_{i}}\right)^{4 / 3}\right]^{-1} .
$$

Using $G_{p v}(j \omega)$ given by (19), the spectral density of the wind pressure, $S_{p}(\omega)$, is calculated by

$$
S_{p}(\omega)=G_{p v}(j \omega) \sqrt{S_{v}(\omega)} C(\omega) \sqrt{S_{v}(\omega)} G_{p v}^{*}(j \omega) .
$$

\subsection{Transfer Function Matrix $G_{p w}(j \omega)$}

Write the constant spectral density of the seed of randomness $w(t)$ as $S_{w}(\omega)=W(>0)$. Then, $S_{p}(\omega)$ is given by (see Fig. 1)

$$
S_{p}(\omega)=G_{p w}(j \omega) W G_{p w}^{*}(j \omega) .
$$

To compare (22) with (21), decompose the coherence matrix $C(\omega)$ into upper and lower matrices as

$$
C(\omega)=L(\omega) U(\omega) .
$$

Then, the comparison yields

$$
G_{p w}(j \omega) W^{\frac{1}{2}}=G_{p v}(j \omega) \sqrt{S_{v}(\omega)} L(\omega)
$$


or

$$
G_{p w}(j \omega)=G_{p v}(j \omega) \sqrt{S_{v}(\omega)} L(\omega) W^{-\frac{1}{2}}
$$

Consequently, the transfer function matrix $G_{p w}(j \omega)$ from the computer-simulated white noise $w(t)$ to the wind pressure $p(t)$ can be given by (24) with (12), (19) and (23), and hence the impulse response matrix $H_{p w}(t)$ can be computed by the formula (5). From (24) we know indirectly that $G_{v w}(j \omega)$ is given as

$$
G_{v w}(j \omega)=\sqrt{S_{v}(\omega)} L(\omega) W^{-\frac{1}{2}}
$$

The matrix $W$ is a user-defined parameter, so we set as $W=I_{n}$ (unit matrix of dimension $n$ ) for simplicity in the sequel.

\section{Estimation of Wind Load Spectrum}

In the previous section, we have proposed a method of generating wind loads artificially from the white noise simulated by a digital computer. In this section, we shall consider the problem of estimating wind load from dynamic data of the output of building structure. We shall investigate this problem by simulation studies using artificial wind loads generated in the previous section.

As for the high-rise building structures, they are modeled traditionally, in most cases, as a multi-degree-offreedom system based on the shear model in which only the shear deformation is considered. It is wellknown, however, that the bending deformation becomes so large that it can not be neglected but must be taken into consideration positively whenever we consider the control of high-rise buildings more than fifty stories or equivalent to $200[\mathrm{~m}]$ in height. Because the motion due to bending affects severely the habitability or comfortable feeling for people in the building, the bending motion should be taken into account in the control and/or stability analysis of the high-rise buildings. In order to control the vibrations of such buildings, a rather more sophisticated model called the bendingshear model (BS model) will be appropriate to describe its dynamics. In this section, the BS model is adopted.

Suppose an $n$-story high-rise building. Let $\left\{u_{i}(t)\right\}$ and $\left\{\theta_{i}(t)\right\}(i=1,2, \cdots, n)$ be transverse displacement (shearing) and rotation angle (bending) of the $i$ th floor (see Fig.2). Then, according to the BS model of the structure the dynamics are described by the following $2 n$-dimensional vector differential equation:

$$
M \ddot{z}(t)+D \dot{z}(t)+K z(t)=B_{0} p(t)
$$

in which $z(t)=\left[u^{T}(t), \theta^{T}(t)\right]^{T}\left(u(t)=\left[u_{1}(t), \cdots\right.\right.$, $\left.\left.u_{n}(t)\right]^{T}, \theta(t)=\left[\theta_{1}(t), \cdots, \theta_{n}(t)\right]^{T}\right) ; p(t)=\left[p_{1}(t)\right.$, $\left.p_{2}(t), \cdots, p_{n}(t)\right]^{T}$ is the wind load; $M, D$ and $K$ are $2 n \times 2 n$-dimensional mass, damping and stiffness matrices, respectively; and $B_{0}$ is a $2 n \times n$-matrix. The detail
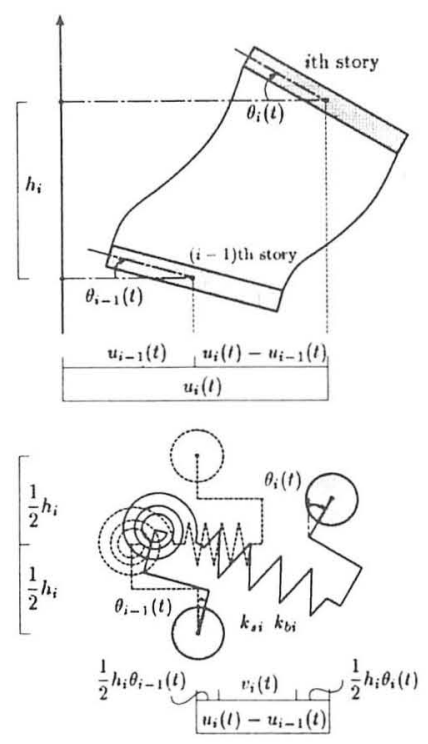

Fig. 2: Bending-shear model.

derivation of the dynamics (26) will appear elsewhere [13].

Assume that we are given data on $z(t)$ as the observation data:

$$
y(t)=C_{0} z(t)
$$

where $C_{0}$ is an $n \times 2 n$-matrix. Based on the model (26) the reduced-order model can be derived from the model point of view. However, the estimation of the power spectral density of the random load using such a reduced-order model was computationally unstable because the inverse of the frequency transfer function matrix from $p(t)$ to the output $y(t)$ exhibits a computational singularity. So, we take rather a more straight forward approach.

Laplace-transforming (26) and (27), we get

$$
Y(s)=G_{0}(s) P(s),
$$

where $Y(s)=\mathcal{L}[y(t)], P(s)=\mathcal{L}[p(t)]$, and

$$
G_{0}(s)=C_{0}\left(s^{2} M+s D+K\right)^{-1} B_{0} .
$$

Hence, the power spectral density of the output $y(t)$, $S_{y}(\omega)$, is given by

$$
S_{y}(\omega)=G_{0}(j \omega) S_{p}(\omega) G_{0}^{*}(j \omega)
$$

in which $S_{p}(\omega)$ is the power spectrum to be estimated of the wind load $p(t)$. Then, the power spectrum $S_{p}(\omega)$ can be computed from $S_{y}(\omega)$ by

$$
S_{p}(\omega)=G_{0}^{-1}(j \omega) S_{y}(\omega) G_{0}^{-*}(j \omega),
$$

where $G_{0}^{-*}(\omega)=\left[G_{0}^{*}(j \omega)\right]^{-1}$. 

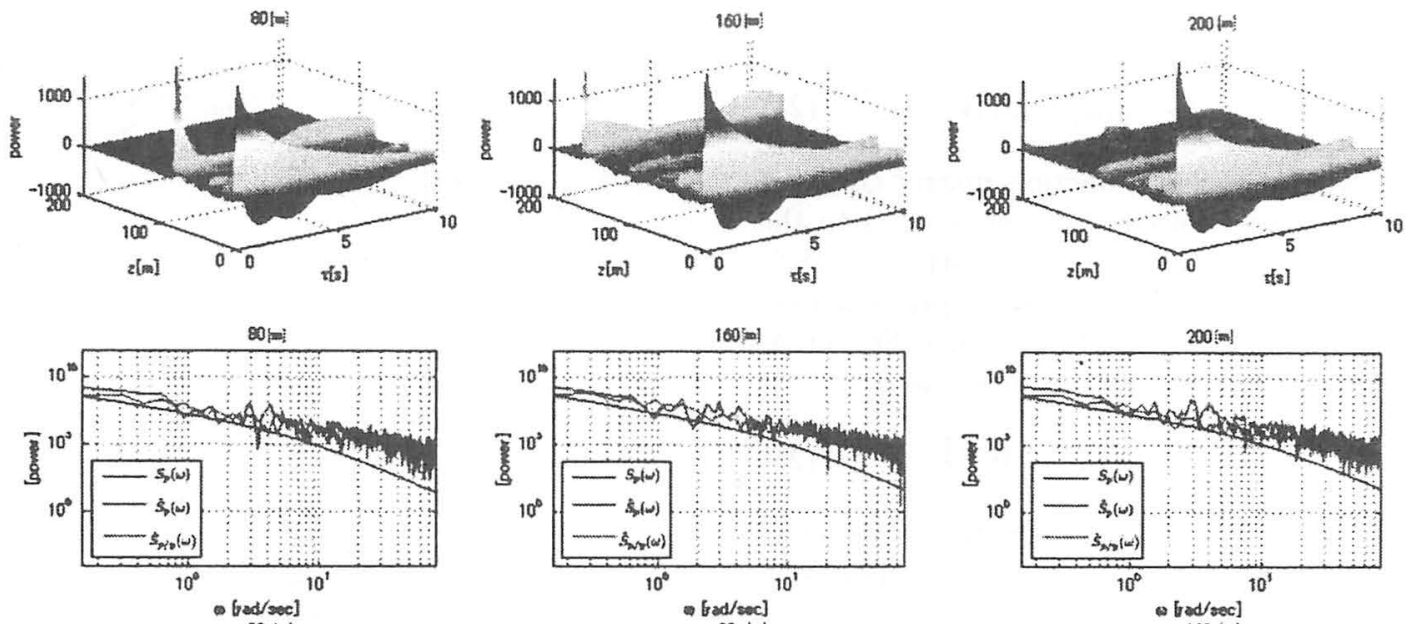

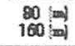
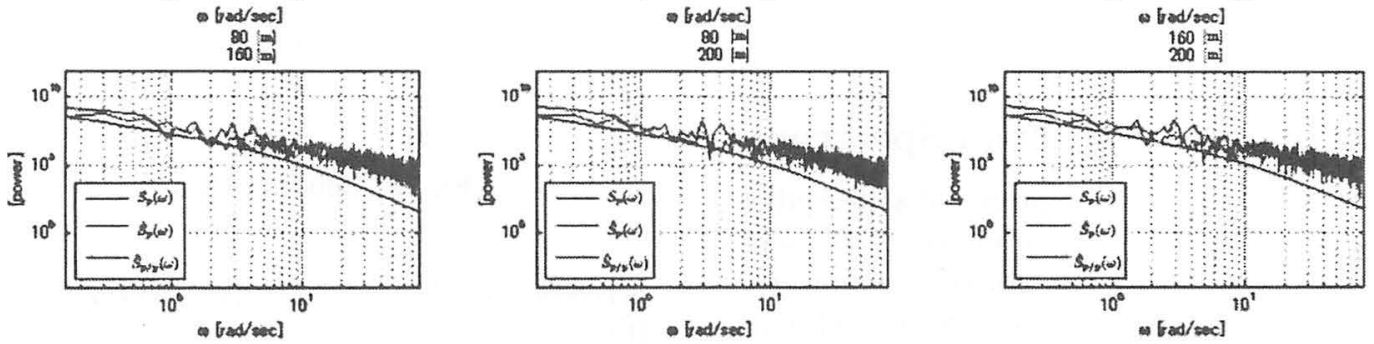

Fig. 3: Unit impulse response, power spectra and cross power spectra.

\section{Simulation Studies}

The aim of this section is twofold:(i) to show how well the random wind loads can be generated from the computes-simulated white noise processes, and (ii) to confirm that the proposed method in Section 3 is useful to estimate the spectrum of the wind load affecting the building. A fifty-story building with $4[\mathrm{~m}]$ floor-height is assumed in the simulation studies.

Top three figures in Fig. 3 illustrate impulse responses $\left\{h_{p_{i} w_{k}}(t)\right\}$ from the simulated white noise to the generated wind pressure $p_{i}(t)$ for $i=20,40$ and 50. The spectral densities are depicted in the lower part of the figure. The smooth curve which is labelled by $S_{p}(\omega)$ shows the theoretical density given by (22); while $\hat{S}_{p}(\omega)$ is the density obtained by the sample path of the wind pressure $p(t)$ which is numerically generated from (4) using impulse responses $\left\{h_{p_{i} w_{k}}(t)\right\}$. In Fig. 3 , though $\hat{S}_{p}(\omega)$ seems to take somewhat higher values than the theoretical $S_{p}(\omega)$ over the whole region, it may be said from the architectural point of view that these two coincide with each other, especially, in the range of $10^{0}-10^{1.2}$ which covers the frequencies corresponding from the first to the fifth modes of the relevant building structure. As a result, we may say that the wind loads are generated numerically fairly well.

The spectral density of the unknown wind loads, $\hat{S}_{p / y}(\omega)$, which is estimated from the observation data taken for the structure output $y(t)$ is also depicted in Fig. 3. In order to obtain the observation data $y(t)$, the same wind load $p(t)$ generated to obtain $S_{p}(\omega)$ was used. Comparison of $\hat{S}_{p / y}(\omega)$ and $\hat{S}_{p}(\omega)$ shows fairly good agreement. In this sense, the proposed method in Section 4 seems to work well.

\section{Concluding Remarks}

The method of generating random wind load proposed in this paper is rather conveniently feasible than that proposed in [8], [9], so that it will be a useful tool for the analysis of the wind-induced response of the building structure because the statistics used in constructing impulse response matrix are quite free to choose in adapting to desired ones.

The approach to estimate the unknown wind load proposed in Section 3 may seem somewhat brute-force because its depends merely on the Fourier-inversion. However, it should be remembered in mind that this approach yields certainly a successful result.

\section{References}

[1] A. Ohsumi and A. Ueda, Modeling of seismic waves using chirp signals and simulations, Int. J. Innovative Computing, Information and Control, vol. 2, no. 5, pp. 1053-1063, 2006.

[2] E. Simiu and R. H. Scanlan, Wind Effects on Structures; An Introduction to Wind Engineering, John Wiley, New York, 1978. 
[3] A. G. Davenport, Past, present and future of wind engineering, J. of Wind Engineering (Proc. 5th Asia-Pacific Conf. on Wind Engineering, Kyoto, 2001), no. 89, pp. 1-8, 2001.

[4] G. Solari, Analytical methods for estimating the wind-induced response of structures, $J$. of Wind Engineering (Proc. of 5th Asia-Pacific Conf. on Wind Eng., Kyoto, 2001), no. 89, pp. 45-54, 2001.

[5] Z. Hui, A. Bachmann, and C.-A. Graubner, Wind loads on high-rise structures - Comparative study of code provisions according to Eurocode 1 and Chinese codes, J. of Wind Engineering (Proc. of 5th Asia-Pacific Conf. on Wind Engineering, $\mathrm{Ky}-$ oto, 2001), no. 89, pp.613-616, 2001.

[6] Y. Tamura, Wind excitations and mitigation of vibrations, Proc. of 2000 Symp. on Passive Control of Structures, pp. 37-46, Tokyo, Mar. 2000 (in Japanese).

[7] Architectural Institute of Japan (AIJ): Structural Design Concepts for Earthquake and Wind, Maruzen, Tokyo, 1999 (in Japanese).

[8] Y. Iwatani, Simulation of multidimensional wind fluctuations having any arbitrary power spectra and cross spectra,, J. of Wind Engineering, no. 11, pp. 5-18, 1982 (in Japanese).

[9] Y. Iwatani, Simulation of multidimensional wind fluctuations associated with given power spectra and cross spectra and its accuracy, J. of Wind Engineering, no. 36, pp.11-26, 1988 (in Japanese).

[10] J. Minato and A. Ohsumi, Modeling of wind profile gratifying given power and crossspectra, Int. J. of Innovative Computing, Information and Control, vol.6, no.3, 2010 (to appear).

[11] A. G. Davenport, The dependence of wind loads on meteorological parameters, Proc. Int. Seminar of Wind Effects on Buildings and Structures, Ottawa, pp. 19-82, 1967.

[12] Architectural Institute of Japan (AIJ): AIJ Recommendations for Loads on Buildings (English Edition), Maruzen, Tokyo, 1996.

[13] J. Minato, A. Ohsumi, and Y. Sawada, Artificial generation of wind load and estimation of wind disturbance affecting on high-rise buildings given by bending-shear model [tentative], in preparation. 